\title{
MODELIZACIÓN GEOESPACIAL DEL ESCURRIMIENTO SUPERFICIAL EN CUENCAS DE LAS SIERRAS DEL SUDOESTE, TUCUMÁN, ARGENTINA
}

\section{GEOSPATIAL MODELING OF SURFACE RUNOFF IN WATERSHEDS OF THE SOUTHWEST HILLS, TUCUMÁN, ARGENTINA}

\author{
Romina Díaz Gómez ${ }^{1,2}$ y Fernanda Julia Gaspari ${ }^{1}$ \\ ${ }^{1}$ Curso de Manejo de Cuencas Hidrográficas. Facultad de Ciencias Agrarias y \\ Forestales, Universidad Nacional de La Plata. Diagonal 113 N 469. La Plata, \\ Argentina. Tel. 0221-423-6616 \\ ${ }^{2}$ CONICET, CCT La Plata. \\ E-mail de contacto: rominadiazgomez@gmail.com
}

Díaz Gómez, R. \& Gaspari, F. J. (2017). Modelización geoespacial del escurrimiento superficial en cuencas de las sierras del Sudoeste, Tucumán, Argentina. Revista Estudios Ambientales, 5(1), 29-47. 


\section{RESUMEN}

La aplicación del modelo hidrológico L-THIA @, con apoyo en la metodología del número de la curva $(\mathrm{CN})$ del Servicio de Conservación de Suelos de Estados Unidos, es empleado para transformar la precipitación total en precipitación efectiva, constituyéndose en una herramienta de gran valor para realizar estudios hidrológicos en cuencas hidrográficas, en las que no se cuenta con registros lo suficientemente extensos y confiables. Esta metodología requiere del conocimiento del tipo y uso de suelo de la cuenca en estudio, así como de registros de precipitación, en estaciones cercanas a ella. El presente estudio se aplica en la cuenca hidrográfica de los ríos Singuil y Chavarria, Tucumán, Argentina. Se cuantificó el uso y cobertura del suelo a partir del procesamiento de imágenes Landsat TM, identificando los cambios de uso y cobertura del suelo para el período 1986-2010. El procesamiento digital de la base de datos vectorial consistió en la rasterización automática con herramientas de sistema de información geográfica. Se obtuvo el valor de $\mathrm{CN}$ y se cuantificó la lámina de escurrimiento. La disminución de la cobertura de pastizal y su reemplazo por bosque nativo, incrementa la tasa de infiltración reduciendo el escurrimiento superficial.

Palabras clave: suelo, número de curva, L-THIA @, sierras del sudoeste, Tucumán.

\section{ABSTRACT}

The application of the L-THIA @, hydrologic model, supported on the curve number methodology $(\mathrm{CN})$, SCS-USA, it is used to transform total precipitation into effective precipitation. This becomes a useful tool for hydrologic studies in basins lacking extended and truthful registers. This methodology requires: type of soil data, land use land cover map, and precipitation data. The study area was Singuil and Chavarria basins, Tucumán, Argentina. We analyzed land use and cover change during 19862010 using Landsat TM images. Vectorial data base was rasterized using Geographic Information System. CN value and run off level were obtained.The decrease of grassland cover and its replacement by native forest increases the rate of infiltration reducing the surface runoff in the analyzed basins.

Keywords: soil, curve number, L-THIA @ , sierras del sudoeste, Tucumán. 


\section{INTRODUCCIÓN}

Los cambios de uso y cobertura del suelo presentan una compleja relación con la dinámica hídrica en una cuenca hidrográfica, ya que influye en la intercepción de la lluvia, retardo de la escorrentía y aumento de la infiltración, regulando de este modo el rendimiento hídrico de la cuenca y evitando crecientes e inundaciones (Mármol, 2006), causando impactos tanto en el comportamiento hidráulico como hidrológico.

En Argentina, en la provincia de Tucumán, en las cuencas de las Sierras del Sudoeste, se encontró que el proceso de transición forestal está presente en la cuenca del río Marapa, debido principalmente a la expansión de bosques en las subcuencas de los ríos Singuil y Chavarría (Díaz Gómez, 2015). La disminución de la población rural, seguramente se asocia con la disminución del ganado observado en la zona de montaña. La disminución de la este factor de presión, permite la regeneración vegetal en zonas de montaña (Ayarde y González, 2013).

Estos estudios constituyen otro caso de recuperación de ecosistemas en América Latina. La trayectoria de los cambios de uso / cobertura de la tierra observados, es similar a la ocurrida en países desarrollados (Mather y Needle, 1998) y algunas regiones tropicales (Grau et al., 2003; Klooster, 2003; Redo et al., 2012), donde ocurren procesos de transición Forestal, asociados a cambios socioeconómicos (Díaz Gómez, 2015).

Los modelos hidrológicos son una herramienta útil para la evaluación y análisis de los cambios de uso del suelo y su influencia sobre los recursos hídricos. Constan de entradas y salidas que consisten en variables hidrológicas mensurables conectadas por medio de ecuaciones (Chow, et al. 1994). Al ser un representación incompleta de la realidad, se debe definir de manera clara el objetivo para el cual es aplicado, la exactitud de la repuesta, las simulaciones y las variables de entrada que utiliza (según disponibilidad de base datos y costo y tiempo para su relevamiento).

Una de las variables hidrológicas más importante en el análisis de la dinámica hídrica y su modelización es el escurrimiento superficial, considerado como el agua procedente de la lluvia que circula por la superficie y se concentra en los cauces. Su distribución temporal y espacial es función de las características topográficas, geológicas, climáticas y de vegetación de la cuenca y está íntimamente ligado a la relación entre aguas superficiales y subterráneas (Gaspari et al., 2009). 
El modelo hidrológico más generalizado y fácil de adaptar a cualquier región geográfica es el método del número de curva $(\mathrm{CN})$. Este método, desarrollado por el Soil Conservation Service (SCS) de Estados Unidos (1972), se basa en la estimación directa del escurrimiento superficial de una lluvia aislada a partir de características del suelo, uso del mismo y de su cubierta vegetal (Gaspari et al., 2015).

La modelización hidrológica del NC, bajo entorno geoespacial con Sistemas de Información Geográfica (SIG), se formula en el modelo L-THIAC (Long-Term Hydrologic Impact Assessment and Non Point Source Pollutant Model) (Harbor, 1994) que se ha ejecutado, por primera vez en Argentina, en dos cuencas hidrográficas de la provincia de Buenos Aires, con el fin de comprobar la viabilidad de utilizar el modelo en cuencas hidrográficas rurales del país (Vázquez Amabile et al., 2008).

El modelo L-THIA @ (Harbor, 1994) fue desarrollado por la universidad de Purdue para evaluar como los cambios de uso del suelo afectan el escurrimiento anual a escala de cuenca. Este modelo es empleado para transformar la precipitación total en precipitación efectiva, constituyéndose en una herramienta de gran valor para realizar estudios hidrológicos en cuencas hidrográficas, en las que no se cuenta con registros lo suficientemente extensos y confiables (Delgado, 2012).

En numerosos estudios fue aplicado el L-THIA (c) para proporcionar información sobre los impactos hidrológicos relativos a la dinámica hídrica para diferentes escenarios de uso del suelo, promoviendo un diagnóstico y aproximación a potenciales problemas a largo plazo (Ryu et al., 2001; Guzmán et al., 2010; Berlanga-Robles et al., 2011), por ejemplo en cuencas de llanura de la provincia de Buenos Aires (Delgado, 2012) y en cuencas subtropicales (Duet al., 2012; Sun et al., 2014).

El objetivo del presente trabajo fue modelar la relación precipitación - escurrimiento en cuencas hidrográficas en diferentes escenarios de uso del suelo para el período 19862010 en las Sierras del sudoeste, provincia de Tucumán, Argentina, aplicando el software L-THIA @.

\section{METODOLOGÍA}

Área de estudio

Las cuencas hidrológicas que se encuentran en los faldeos orientales de las sierras del Aconquija y del Sudoeste, conforman un el divortium aquarum que constituye el 
límite provincial entre Tucumán y Catamarca. La Sierra del Aconquija conforma la entidad orográfica de mayor altura de la provincia de Tucumán (Rohmeder, 1949); mientras que, las Sierras del Sudoeste, ubicadas al sur, se dividen en dos brazos: hacia el oeste la Serranía de Santa Ana y hacia el este las Sierras de Narváez, entre ambas se ubica el Embalse de Escaba (Figura 1), creado en 1949 con propósitos múltiples, como ser la atenuación de crecidas, regulación de los caudales naturales para riego, producción de energía hidroeléctrica, y desarrollo de actividades recreativas (Díaz Gómez, 2015).

Las cuencas en estudio son las de los ríos Singuil y Chavarría, ubicadas en las Sierras del Sudoeste, y son el aporte hídrico al Embalse de Escaba.

La cuenca del río Chavarría presenta un área de $257 \mathrm{~km}^{2}$ y la cuenca del río Singuil $550 \mathrm{~km}^{2}$. El rango de elevación oscila entre $685 \mathrm{msnm}$ y $271 \mathrm{msnm}$ en la cuenca del río Chavarría. La cuenca del río Singuil tiene una altura máxima de $2.168 \mathrm{msnm}$ y mínima de 678 msnm. Presentan un clima subtropical monzónico con estación lluviosa en verano y seca en invierno, con una precipitación media anualde1.200 mm; durante el mes más lluvioso (verano) $167 \mathrm{~mm}$ y durante el mes más seco (invierno) $3 \mathrm{~mm}$. La temperatura media anual es de $17,6 \stackrel{\circ}{ } \mathrm{C}$. Se caracterizan por un gradiente ambiental altitudinal, con pastizales de altura y bosques subtropicales en las cabeceras de las cuencas y agricultura en las zonas de baja pendiente (Díaz Gómez, 2015). 


\section{revista estudios ambientales}

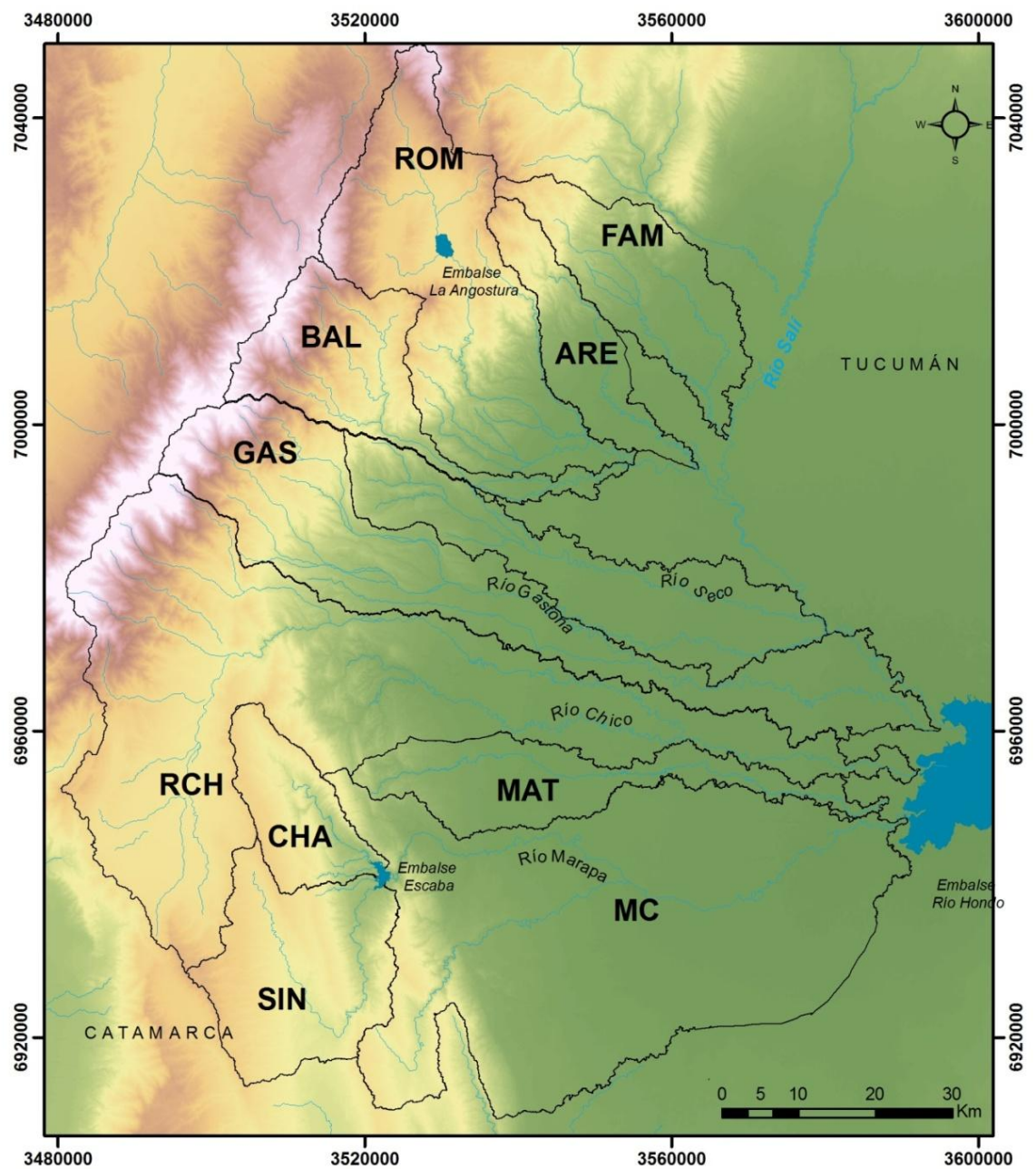

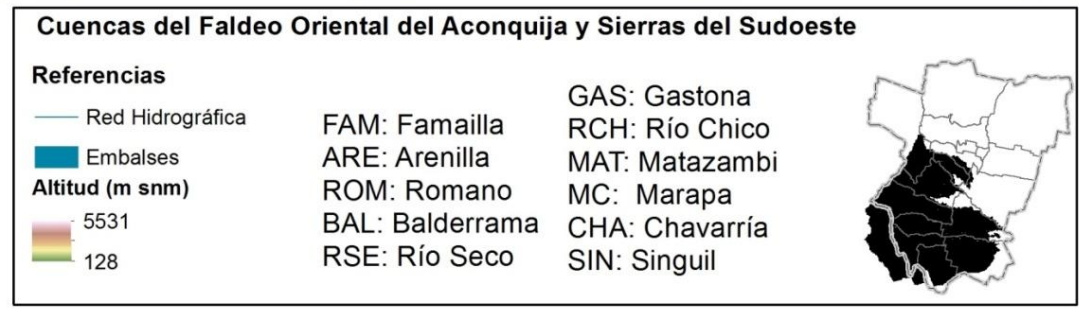

Figura 1. Cuencas hidrológicas del faldeo oriental de las sierras del Aconquija y del sudoeste de la provincia de Tucumán (Díaz Gómez, 2015).

\section{Simulación del escurrimiento superficial: Modelo L-THIA (C)}

Para conocer el efecto del cambio de uso y cobertura del suelo en el escurrimiento superficial en las cuencas de los ríos Singuil y Chavarría durante el período 19862010, se aplicó el modelo L-THIA @ (Harbor, 1994), bajo entorno de un Sistema de Información Geográfica (SIG) fortaleciendo el procesamiento geoespacial y temporal 
de la información. Se basa en el método de número de curva ( $\mathrm{CN}$ ), requiere información cartográfica uso y cobertura del suelo, grupo hidrológico de los suelos y datos de precipitación (Harbor, 1994; Wang et al., 2005; Engel et al., 2003; Sun et al., 2014).

El método de CN del Servicio de Conservación de Suelos (SCS) de Estados Unidos utiliza una ecuación para cuantificar el escurrimiento superficial que se expresa como el cociente entre $(P-l a)^{2}$ y $(P-l a+S)$, donde $P$ es la precipitación, la es la abstracción inicial, $S$ es el máximo potencial de retención de la cuenca ante un evento de tormenta. El S se determina por el cociente entre $(25.400-254 \times \mathrm{CN})$ y CN considerando $\mathrm{CN}$ como el valor de número de curva, representado por la combinación de valores entre el grupo hidrológico de suelo, las clases de coberturas y usos del suelo y la condición de humedad antecedente del suelo. El SCS desarrolló una relación empírica entre la y $S$, siendo la $=0,2 \times S$ (Gaspari et al., 2015). En síntesis, el modelo se basa en la ecuación $\mathrm{Qv}=\mathrm{Qd} \times \mathrm{A}$, donde para la determinación del volumen de escurrimiento $(\mathrm{Qv})$ (en $\mathrm{mm}$ ), parte de la multiplicación de la profundidad de escurrimiento $(Q d)$ y el área de escurrimiento $(A)$.

La aplicación del modelo requiere de cartografía en formato vectorial, codificadas de forma tal que, luego del rasterizado automático, procesamiento y asignación de valores particulares según el caso, se genera un mapa de distribución de número de curva $(\mathrm{CN})$ a partir del cual se determina lámina de escurrimiento, aplicando la metodología del SCS. El modelo cuantifica el escurrimiento superficial en función de el volumen y profundidad de la lámina (Wang et al., 2005) a partir de datos de precipitación.

Para la aplicación del modelo se desarrollaron las siguientes capas de información para las cuencas del río Chavarría y Singuil:

- Mapa de uso y cobertura de suelo realizado a partir de la clasificación de imágenes satelitales Landsat TM (Landsat Thematic Mapper), del 11deseptiembre1986,18agostoy28deagosto2010obtenidas del Instituto Nacional de Pesquisas Espaciais de Brasil (INPE) con un preprocesamiento, y corrección atmosférica Dark Object Substraction y una reproyección a Posgar 98 (Vanonckelen et al., 2013). Posteriormente, mediante el método de clasificación paramétrica definida como Support Vector Machine (SVM), se desarrolló en forma precisa la clasificación de coberturas del suelo (Shao y 
Lunetta 2012). El mapa de cobertura y uso del suelo para el año 2010 de las cuencas Singuil y Chavarría presentaron una precisión total de 89 \% (Díaz Gómez, 2015).

- Mapa de distribución espacial de los suelos según el grupo hidrológico (GH), fue asignado a partir de la textura superficial definida en el Atlas de suelos de la República Argentina del INTA (2005), escala 1:500.000, procesado y reclasificado a una resolución espacial de pixel de $30 \mathrm{~m}$.

- Los registros pluviométricos que se utilizaron se obtuvieron de estaciones meteorológicas ubicadas en las respectivas cuencas y corresponden a datos diarios durante el período 2001-2010, de la Hidroeléctrica Tucumán S.A. Se consideró un evento extremo identificado del analisis bases de datos de precipitación mencionada. Para la cuenca del río Singuil se identificó una tormenta máxima de $46 \mathrm{~mm}$ el día 02/01/2006 y para la cuenca del río Chavarría, la máxima tormenta fue de $60 \mathrm{~mm}$ con igual fecha. La modelación propuesta partió de un supuesto de que el modelo meteorológico es homogéneo en toda la cuenca.

Las tres capas de información fueron adaptadas a las necesidades del modelo para el procesamiento con SIG. A partir de los mapas uso y cobertura del suelo y $\mathrm{GH}$, el modelo estableció los valores de $\mathrm{CN}$ a nivel de píxel, en forma automática. Posteriormente se ejecutó el modelo a fin de evaluar la respuesta hidrológica temporal - espacial de cada cuenca en estudio para una tormenta.

\section{RESULTADOS Y DISCUSIÓN}

Los resultados alcanzados con L-THIA @, permitieron obtener la cuantificación de la lámina de escurrimiento ante eventos extremos de tormenta, para las correspondientes cuencas.

La caracterización edáfica superficial de las cuencas indican que los suelos de grupo hidrológico $D$ (suelos con afloramiento rocosos) y con mayor potencial de escurrimiento, ocupan el $67 \%$ de la cuenca del río Chavarría y $78 \%$ de la cuenca del río Singuil. Mientras que los suelos del grupo hidrológico C, ocupan el $22 \%$ del área en cuenca del río Chavarría y el $33 \%$ en la cuenca del río Singuil, tratándose de 
suelos con textura franco-limosa, cuyo potencial de escurrimiento es menor que el correspondiente a GH D.

La vegetación identificada en las cuencas se conformó por tres grandes clases:

Bosque: conformada por los pisos altitudinales de las yungas australes, dominado por el bosque montano, selva montana y selva pedemontana. De acuerdo a Brown (2002), el bosque está dominado por especies pertenecientes a la Selva del Cebil (Piptademia macrocarpa), Selva de tipa y pacará (Tipuanatipu y Enterolobium contortisiliquum).

Pastizal: constituido por la cobertura natural de pastizales de altura, formada por matas de gramíneas con predominio de Festuca sp.

Cultivo de granos (Agricultura): incluye los cultivos de soja, trigo y suelo desnudo.

La distribución espacio-temporal de la vegetación se expresa en la Figura 2.

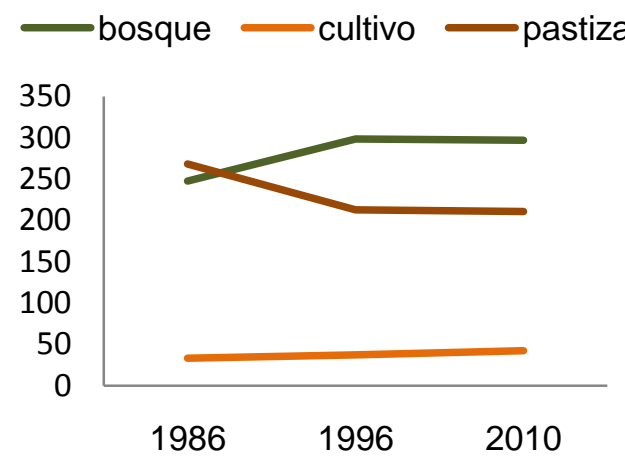

a. Cuenca Chavarría

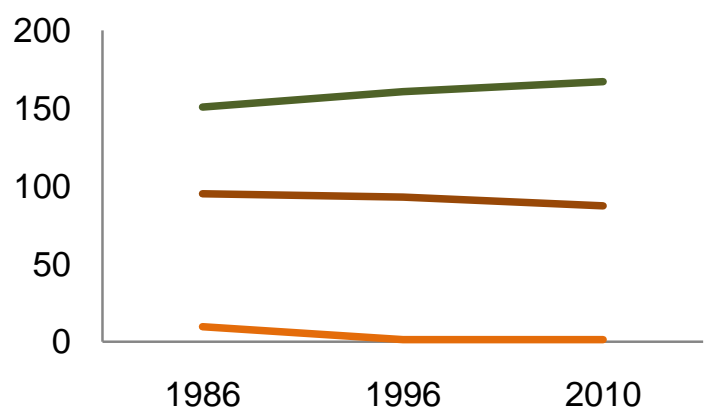

b. Cuenca Singuil

Figura 2. Superficie $\left(\mathrm{km}^{2}\right)$ de uso y cobertura del suelo durante el periodo 1986-2010.

En la Figura 2 se representa la evolución de del análisis espacio temporal de cambio de uso del suelo en las cuencas de las Sierras del Sudoeste, la cual reflejó que la cobertura de bosque incrementó durante el período analizado, desde el $42 \%$ del área en el año 1986 a un 50\% en el año 2010, que corresponde a una ganancia neta de 64 $\mathrm{km}^{2}$, expandiéndose sobre áreas de cultivos abandonadas y sobre la clase pastizal durante el período estudiado. La superficie de pastizal disminuyó el 30\% en total. La superficie de cultivo se expandió sobre el bosque y el pastizal.

Según estos patrones temporales, los principales cambios en el uso del suelo se dieron en la primera década con procesos de deforestación y una importante 
recuperación de los mismos. Mientras que en la última década tendieron a estabilizase, predominando la cobertura de bosque en las subcuencas altas y las áreas con cultivo en la subcuenca baja, siendo la expansión agrícola el principal uso del territorio en el año 2010.

A partir de estos resultados se realizó la modelización hidrológica con L-THIA @ que representó la variabilidad espacial de $\mathrm{CN}$, en las cuencas del río Singuil y Chavarría para el año 2010 (Figura 3).

La modelización generó cartografía de CN que en la cuenca del río Singuil se presentan valores entre 83 - 86, distribuidos en las zonas con mayor pendiente y cubiertas por pastizales de altura y las áreas con valores de CN entre 40 - 60 están ubicadas en las zonas bajas de la cuenca. Mientras que en la cuenca del río Chavarría los valores de $\mathrm{CN}$ de 59 - 69 se distribuyen en la zona cubierta por bosques a pesar de ser áreas con elevada pendiente. En las zonas de desembocadura de los ríos al embalse, se puede observar valores de $85 \mathrm{CN}$, que representan áreas con mayor escurrimiento, las cuales corresponden a las zonas con cultivos en la cuenca del río Singuil. 


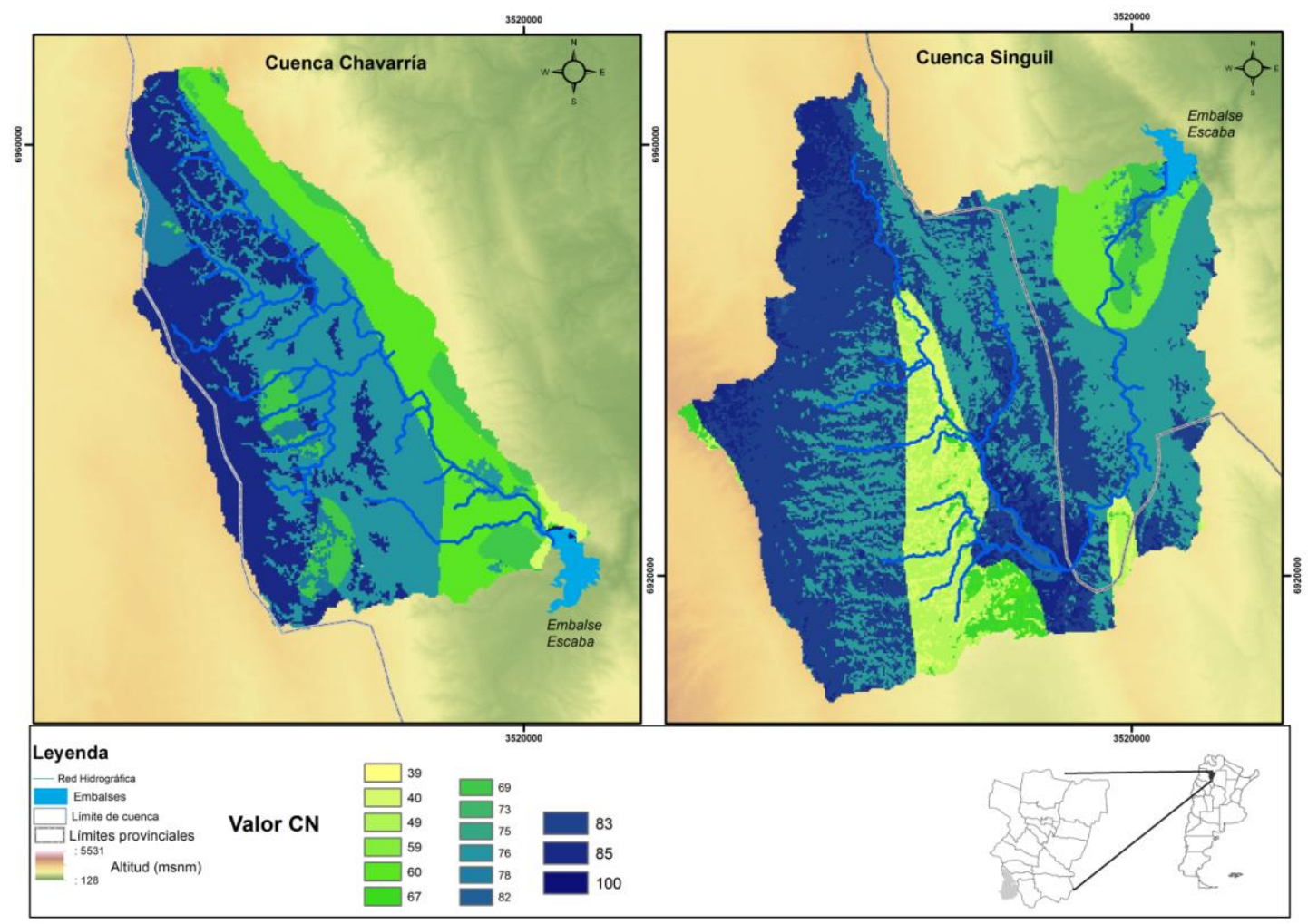

Figura 3. Variabilidad espacial de CN en la cuenca de rio Chavarria (izquierda) y rio Singuil (derecha) en el año 2010.

En la Figura 4 se presentan los mapas de escurrimiento superficial generados por LTHIAC, para el año 2010 ante la tormenta definida para cada cuenca. Se identificaron cambios en los rangos de escurrimiento en ambas cuencas durante el período analizado, que representan la suma total de escurrimiento por cada complejo suelo cobertura y uso del suelo.

Se observa que el volumen de escurrimiento responde espacialmente a la naturaleza de los tipos de suelo presentes.

Los mayores valores de escurrimiento se presentaron en las áreas de pastizales naturales coincidentes con elevadas pendientes (superior a $40 \%$ ) y suelos rocosos. 


\section{revista estudios ambientales}
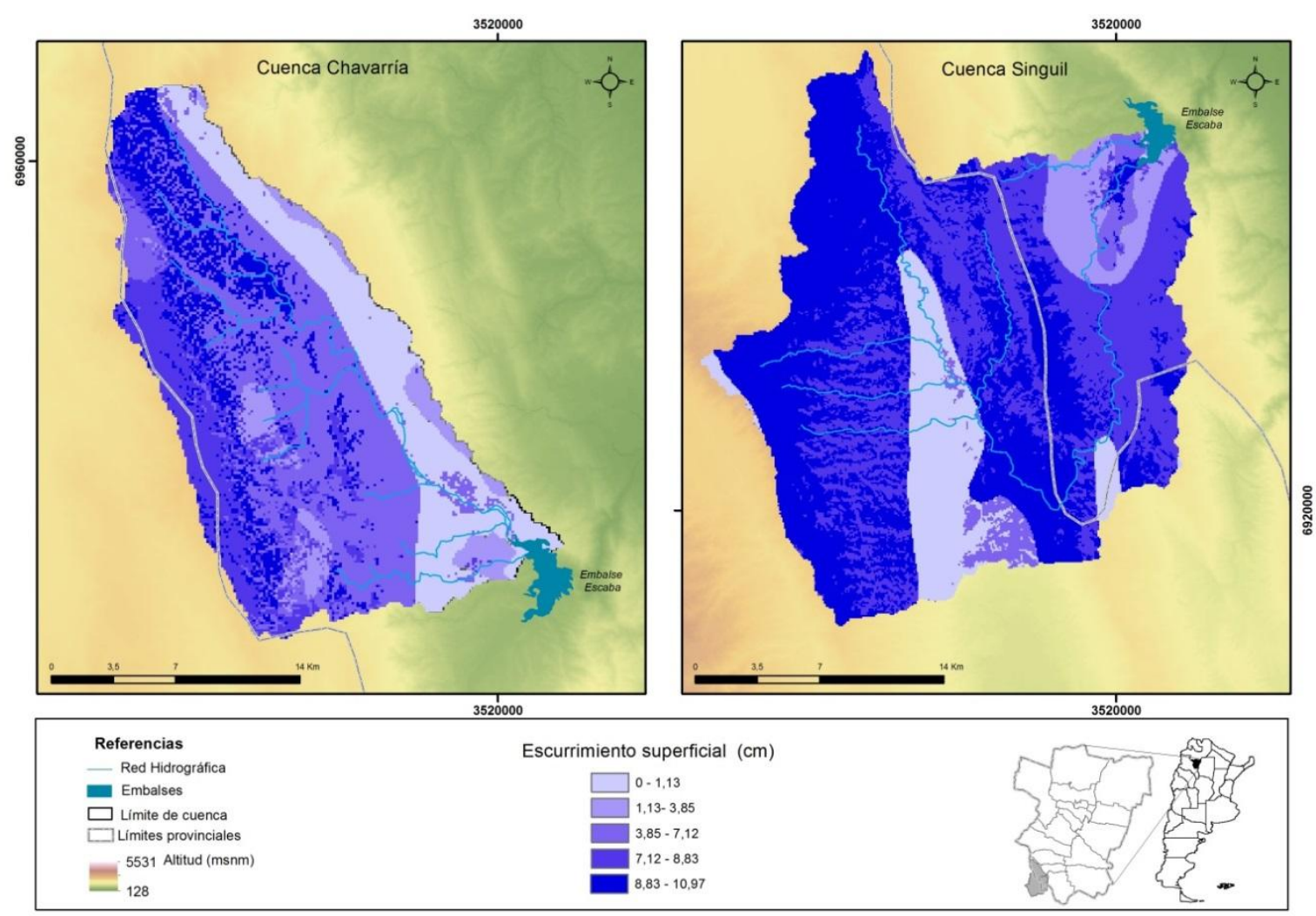

Figura 4. Distribución espacial del escurrimiento superficial para el año 2010 ante una tormenta $60 \mathrm{~mm}$ en la cuenca del río Chavarría (Izquierda) y río Singuil (derecha).

Analizando la zonificación del escurrimiento superficial, se evidencia una coincidencia que en la reducción de zonas con agricultura $\left(8 \mathrm{~km}^{2}\right)$ y la recuperación de superficie boscosa, se redujo el volumen de escurrimiento superficial acumulado a un valor de $2.300 \mathrm{~cm}$ en el año 2010 en la cuenca del río Chavarría. Además, la cobertura de pastizal disminuyó $\left(7 \mathrm{~km}^{2}\right)$ sobre el grupo hidrológico $D$ reduciendo $3.766 \mathrm{~cm}$ de volumen de escurrimiento acumulado en la cuenca en el año 2010 ante una precipitación de $60 \mathrm{~mm}$. Mientras que los máximos rangos de escurrimiento 250-350 $\mathrm{cm}$ disminuyeron y el $66 \%$ de la superficie de la cuenca presenta un rango de escurrimiento superficial acumulado en toda la cuenca entre $200-250 \mathrm{~cm}$ para el año 2010 (Figura 5).

En coincidencia con Díaz Gómez (2015), se encontró que la tasa de infiltración en los pastizales naturales es menor (del 50 \% menos) respecto a la del bosque nativo, calculado a partir de infiltrómetros a campo. 


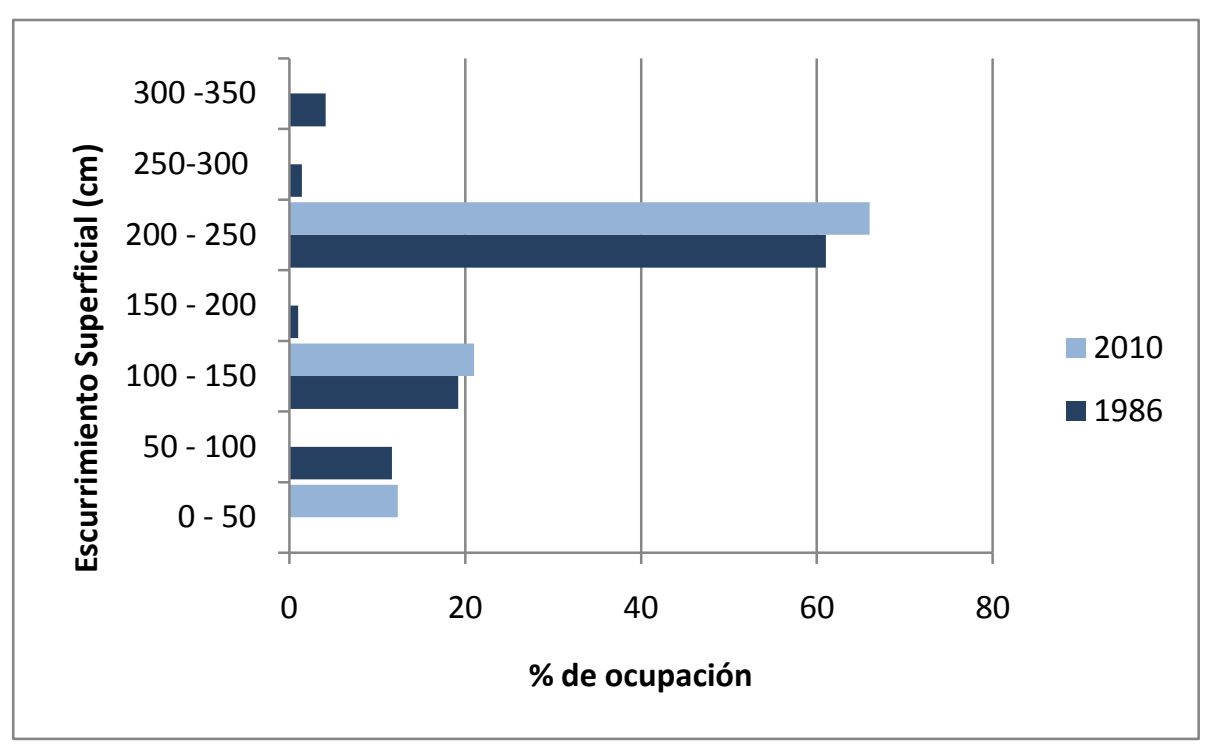

Figura 5. Porcentaje de ocupación en superficie según rangos de escurrimiento acumulado para una tormenta en la cuenca del río Chavarría .

En la Figura 6 se presentan los rangos de escurrimiento superficial acumulado para cada fecha para la cuenca del río Singuil. El rango de escurrimiento entre 150-200 cm incrementó 3 \% en el año 2010 al mismo tiempo que disminuyó 6 \% el área ocupada con rango de escurrimiento entre $200-250 \mathrm{~cm}$.

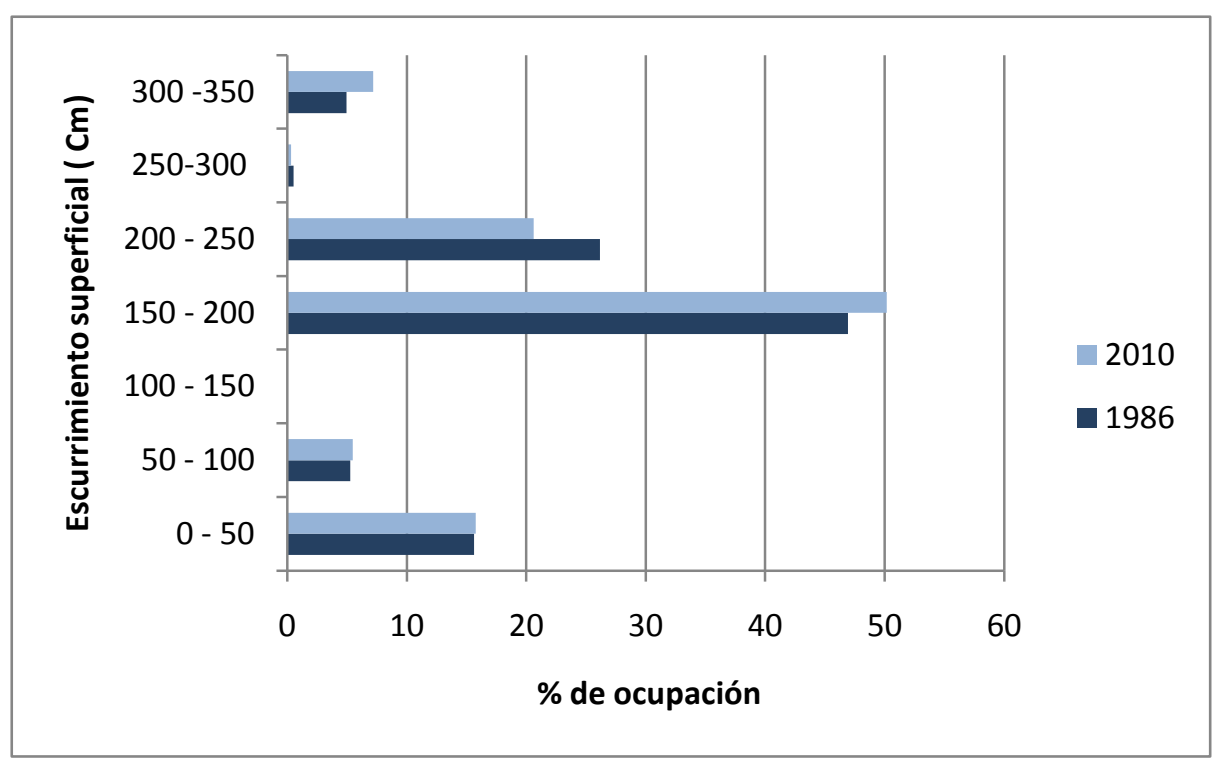

Figura 6. Porcentaje de ocupación en superficie según rangos de escurrimiento superficial acumulado para una tormenta en la cuenca del río Singuil. 


\section{revista estudios ambientales \\ publicación digital del CINEA

Al analizar las diferencias de lámina de escurrimiento total según la cobertura y uso del suelo entre el año 1986 y 2010, los resultados revelan que en la cuenca del río Chavarría, el volumen de lámina de escurrimiento total se reduce $30 \%$ respecto al año 1986, ante una precipitación de $60 \mathrm{~mm}$. Esta situación podría explicarse debido a que hay una pérdida de superficie agrícola lo cual genera una reducción del 36 \% de la lámina de escurrimiento $(1.163 \mathrm{~cm})$ en el año 2010. Además, la expansión del bosque sobre la superficie de pastizal en los suelos de tipo D en al año 2010, explica la reducción total del $32 \%(4.000 \mathrm{~cm})$ de lámina de escurrimiento ante el mismo evento en el año 1986 (Figura 7).

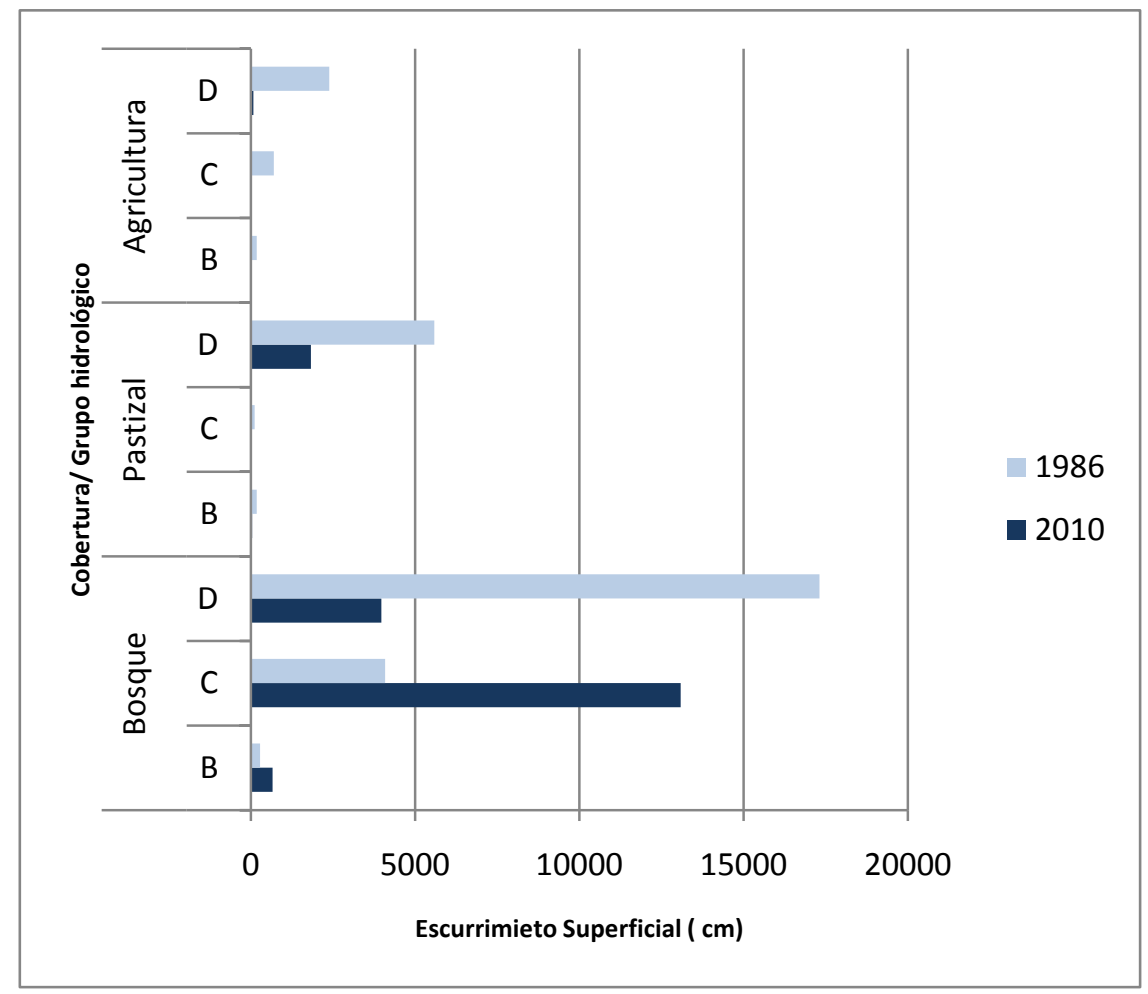

Figura 7. Volumen de escurrimiento superficial acumulado según uso y cobertura del suelo en la cuenca Chavarría, entre 1986-2010.

En la cuenca del río Singuil, la reducción de la cobertura de pastizal $\left(57 \mathrm{~km}^{2}\right)$, impactó negativamente sobre el volumen de escurrimiento, disminuyendo su lámina $22 \%$ $(1.712 \mathrm{~cm})$ en el año 2010. Sin embargo, hay un incremento del volumen de escurrimiento en áreas con avance de agricultura sobre suelos de grupo hidrológico D, de apenas $5 \%(1.307 \mathrm{~cm})$ de lámina. En conjunto, la respuesta hidrológica de la 
cuenca Singuil no cambia significativamente entre 1986 -2010 ante el mismo evento de precipitación (Figura 8).

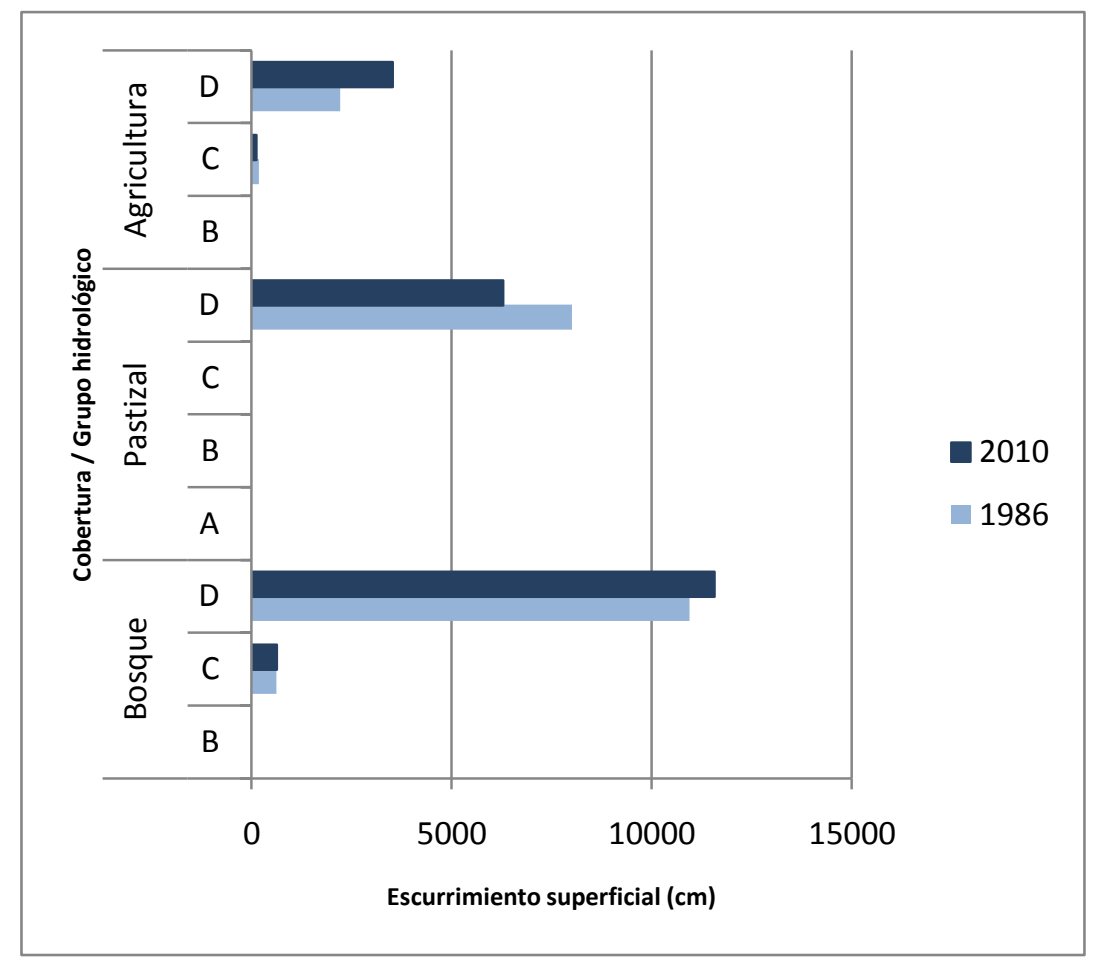

Figura 8. Volumen de escurrimiento superficial acumulado según uso y cobertura del suelo en la cuenca del río Singuil, entre 1986-2010.

Los resultados proporcionan información sobre el cambio en la dinámica hídrica superficial como consecuencia de los cambios espacio-temporales de uso del suelo en las cuencas analizadas. La modelación con L-THIA Cpermitió establecer una zonificación espacial de la lámina de escurrimiento promedio anual caracterizando cada área de las cuencas.

\section{CONCLUSIONES}

La regeneración del bosque sobre el pastizal en las cuencas tendría como consecuencia una mejor regulación de captación del recurso hídrico en las mismas y reducción de inundaciones durante el verano. 
El proceso de transición forestal en las cuencas de las Sierras del Sudoeste influenció en la variabilidad temporal y espacial del escurrimiento superficial. Por lo tanto, la disminución de esta cobertura y su reemplazo por bosque nativo, incrementa la tasa de infiltración reduciendo el escurrimiento superficial.

En las subcuencas del río Singuil y Chavarría se produjo una expansión aproximada de $64 \mathrm{~km}^{2}$ de bosque sobre el pastizal durante el período 1986-2007. Esta situación podría generar un incremento en el servicio ecosistémico de regulación hídrica en las zonas alta de las cuenca, sin embargo el reemplazo total de pastizales por bosque podría generar impactos en la biodiversidad que aún no fueron evaluados.

Este estudio sugiere que las cuencas analizadas son vulnerables a los cambios del uso del suelo, debido a que más del $50 \%$ de su superficie presenta suelos correspondiente al grupo hidrológico D (suelos con afloramiento rocosos) y con mayor potencial de escurrimiento.

La modelización de la relación precipitación - escurrimiento en cuencas hidrográficas en diferentes escenarios de uso del suelo para el período 1986-2010 en las Sierras del Sudoeste, en la provincia de Tucumán, Argentina, aplicando el software LTHIA fue apropiado para estimar y zonificar la influencia del uso del suelo y de las características edáficas superficiales en la respuesta hidrológica. Los resultados que expresa el modelo pueden ser utilizados como información base para futuros análisis ecohidrológicos a menor escala espacial.

Es importante destacar que analizar la respuesta hidrológica de una cuenca hidrográfica de manera geoespacial constituye la base para la planificación del ordenamiento territorial y el desarrollo de planes de manejo integral de cuencas hidrográficas, particularmente en áreas donde la información es escasa.

\section{BIBLIOGRAFÍA}

Ayarde, H., González, J. A. (2013). Recuperación de la vegetación natural por exclusión de pastoreo en una zona de montaña del noroeste de Argentina. Lilloa, 50 (2), 37-49.

Berlanga-Robles, C. A., Ruiz-Luna, A., Covarrubias-Legaspi, H. O., HernándezGuzmán, R. (2011). Patrones de escurrimiento en la Cuenca LechuguillaOhuira-Navachiste, Sinaloa, asociados a cambios de uso del suelo: Una 
aproximación con el modelo del número de curva. Boletín de la Sociedad Geológica Mexicana, 63(1), 39-52.

Brown, A. D., A., Grau, T., Lomáscolo, N. I., Gaspari. (2002). Una estrategia de conservación para las selvas subtropicales de montaña (yungas) de argentina. Ecotropicos, 15(2), 147-159.

Chow, V. T., Mays, D. R., Te Chow, L. W. V., Maidment, D. R., Mays, L. W. (1994). Hidrología aplicada. McGraw-Hill.

Delgado, M. I. (2012). Comportamiento hidrológico en ambientes serranos. Estudio de caso cuenca del Arroyo Belisario. Provincia de Buenos Aires. Tesis inédita Doctorado en la Fac.Cs.Exactas, Ingeniería y Agrimensura. Universidad Nacional de Rosario. (Res. 527/09). 179 pp.

Díaz Gómez, A. R. (2015). Análisis de la vulnerabilidad a cambios climáticos y de uso del territorio de las cuencas hidrológicas del faldeo oriental de la sierra del Aconquija y del Sudoeste, Tucumán. Tesis inédita. Doctorado en Ciencias Biológicas. Universidad Nacional de Tucumán. (Res. № 138/12). 231 pp

Du, J., Qian, L., Rui, H., Zuo, T., Zheng, D., Xu, Y., Xu, C. Y. (2012). Assessing the effects of urbanization on annual runoff and flood events using an integrated hydrological modeling system for Qinhuai River basin, China. Journal of Hydrology, 464, 127-139.

Engel, B. (2003). Long-term hydrologic impacts assessment and non point source pollutant model. Version-2.3 Manual, 7.

Gaspari, F. J., Senisterra, G. E., Delgado, M. I., Rodríguez Vagaría, A., Besteiro, S. (2009). Manual de Manejo Integral de Cuencas Hidrográficas. Primera Edición. La Plata: Universidad Nacional de La Plata. $321 \mathrm{pp}$.

Gaspari, F. J., Senisterra, G. E. y A. R. Díaz Gómez. (2015). Modelización hidrológica con L-THIA en la Cuenca Alta del Arroyo Napaleofú, Buenos Aires, Argentina. XXV Congreso Nacional del Agua. Paraná. Argentina. 8 p.

Grau, H. R., Aide, T. M., Zimmerman, J. K., Thomlinson, J. R., Helmer, E., Zou, X. (2003). The ecological consequences of socioeconomic and land-use changes in postagriculture Puerto Rico. AIBS Bulletin, 53(12), 1159-1168. 
Guzmán, R. H., Ruiz-Luna, A., Berlanga-Robles, C. A., Vekerdy, Z. (2010). Evaluation of total runoff for the Rio San Pedro sub-basin (Nayarit, Mexico) assessing their hydrologic response units. Journal of Spatial Hydrology, 9(2).

Harbor, J. M. (1994). A practical method for estimating the impact of land-use change on surface runoff, groundwater recharge and wetland hydrology. Journal of the American Planning Association, 60(1), 95-108.

Klooster, D. (2003). Forest transitions in Mexico: institutions and forests in a globalized countryside. The Professional Geographer, 55(2), 227-237.

Mármol, L. A. (2006). Introducción al Manejo de Cuencas Hidrográficas y Corrección de Torrentes. Universidad Nacional de Salta. Facultad de Ciencias Naturales.Ingeniería en Recursos Naturales y Medio Ambiente. 287 p.

Mather, A. S., Needle, C. L. (1998). The forest transition: a theoretical basis. Area, 30(2), 117-124.

Redo, D. J., Grau, H. R., Aide, T. M., Clark, M. L. (2012). Asymmetric forest transition driven by the interaction of socioeconomic development and environmental heterogeneity in Central America. Proceedings of the National Academy of Sciences, 109(23), 8839-8844.

Rohmeder, G. (1949). Bosquejo fisiogeográfico de Tucumán. Tucumán: Universidad Nacional de Tucumán. 6p.

Ryu, N. H., Choi, Y. H., Choi, J. D., Choi, B. Y. (1998). The Development of a Javabased, Object-Oriented Modeling System for Simulation of Southern African Hydrology. In 2001 ASAE Annual Meeting (p. 1). American Society of Agricultural and Biological Engineers.

Shao, Y., Lunetta, R. S. (2012). Comparison of support vector machine, neural network, and CART algorithms for the land-cover classification using limited training data points. ISPRS Journal of Photogrammetry and Remote Sensing, 70, 78-87.

Sun, Z., Li, X., Fu, W., Li, Y., Tang, D. (2014). Long-term effects of land use/land cover change on surface runoff in urban areas of Beijing, China. Journal of Applied Remote Sensing, 8(1), 084596-084596. 
Vazquez-Amabile, G. G., Mercuri, P. A., Gaspari, F. J., Engel, B. A. (2008). Construction of a Digital Hydrologic Soil Group Map for Argentina to Simulate Runoff Using GIS-Hydrologic Models. In 21st Century Watershed Technology: Improving Water Quality and Environment Conference Proceedings, 29 March3 April 2008, Concepcion, Chile (p. 83). American Society of Agricultural and Biological Engineers.

Vanonckelen, S., Lhermitte, S., Van Rompaey, A. (2013). The effect of atmospheric and topographic correction methods on land cover classification accuracy. International Journal of Applied Earth Observation and Geoinformation, 24, 921.

Wang, Y., Choi, W., Deal, B. M. (2005). Long-term impacts of land-use change on nonpoint source pollutant loads for the St. Louis metropolitan area, USA. Environmental Management, 35(2), 194-205. 